The Astrophysical Journal, 187:L127-L130, 1974 February 1

(C) 1974. The American Astronomical Society. All rights reserved. Printed in U.S.A.

\title{
SODIUM EMISSION FROM IO: IMPLICATIONS
}

\author{
Michael B. McElroy, Yuk Ling Yung, and Robert A. Brown \\ Center for Earth and Planetary Physics, Harvard University \\ Received 1973 November 29
}

\begin{abstract}
The surface of Io may be covered with a layer of ammonia ice containing trace amounts of sodium, potassium, and calcium; and atmospheric nitrogen could be formed as a photochemical product of ammonia photolysis. Intense sporadic sodium emission from Io can be excited by collisions involving vibrationally excited nitrogen molecules. These metastable molecules may be formed by electron impact, with electrons energized by an auroral mechanism. In order to account for the intensity ratio of the sodium doublet, it is necessary to invoke scattering in a thick gaseous envelope ejected by Io.

Subject headings: abundances - Jupiter - satellites
\end{abstract}

\section{INTRODUCTION}

Io, the innermost Galilean satellite of Jupiter, is known to emit intense, sporadic radiation in the D1 and D2 lines of atomic sodium (Brown 1973). The lines are broad - an emission temperature as high as $8000^{\circ} \mathrm{K}$ can be estimated from the high-resolution spectrum given by Brown and Chaffee (1974), and observed values for the ratio $\mathrm{D} 2 / \mathrm{D} 1$ of the line intensities range between 1.2 and 1.6, in contrast to the value 2.0 which should apply under optically thin conditions. We shall argue that the sodium radiation is excited by collisions involving vibrationally and perhaps electronically excited nitrogen molecules. Nitrogen could be formed as a photochemical product of ammonia photolysis, with ammonia supplied to the atmosphere by evaporation from a layer of surface ice.

\section{PRODUCTION AND LOSS OF $\mathrm{N}_{2}$}

Evidence for $\mathrm{NH}_{3}$ ice on the surface of Io was summarized by Sinton (1973), who concluded that Io should have a thin atmosphere of $\mathrm{NH}_{3}$, with a surface pressure of $5.5 \times 10^{-8} \mathrm{bar}$. This model provides a ready explanation for the observed posteclipse brightening (Binder and Cruikshank 1964; Cruikshank and Murphy 1973) and also accounts for some puzzling aspects of the thermal recovery at $20 \mu$ (Morrison and Cruikshank 1973).

The reflection spectra of Io taken by Johnson and McCord (1970) are also consistent with $\mathrm{NH}_{3}$ ice. A spectral feature between 0.5 and $0.6 \mu$ can be attributed to dissolved sodium. A feature near $0.8 \mu$ may be due to dissolved potassium, and the sharp decrease in albedo at wavelengths less than $0.5 \mu$ could be explained by dissolved calcium. The absorptions associated with sodium and potassium have been carefully studied in the laboratory by Bosch (1954) and by Hurley, Tuttle, and Golden (1968). We were unable to locate any data for calcium, but would expect a broad absorption in the solid matrix associated with the resonance transition of the gas at $4227 \AA$.
The ideas expressed above are not new. They were in fact anticipated in a remarkable paper by Wildt (1939). $\mathrm{He}$ drew attention to the importance of trace alkali metals in solution with $\mathrm{NH}_{3}$ and emphasized in particular their potential role as a coloring agent for Jupiter's clouds. Following Wildt's lead, we might speculate that Io should exhibit a range of colors associated with the global distribution of surface temperature. The effect may have been observed by Kuiper (1973) who recently reported results from direct color photography of Io.

An $\mathrm{NH}_{3}$ surface pressure of $5.5 \times 10^{-8}$ bar would be expected to provide an important source of $\mathrm{N}_{2}$. The chemistry would be initiated by absorption of sunlight at wavelengths less than $2200 \AA$. Subsequent reactions, both gas-phase and heterogeneous, would lead to inevitable production of $\mathrm{N}_{2}$ and $\mathrm{H}_{2}$, and we estimate that the $\mathrm{N}_{2}$ source strength could be as large as $2 \times$ $10^{11}$ molecules $\mathrm{cm}^{-2} \mathrm{~s}^{-1}$ averaged over Io's surface. A source of this magnitude would lead to a concentration of atmospheric $\mathrm{N}_{2}$ in excess of present observational limits (Smith and Smith 1972) in only 3 years. It seems clear that an efficient escape mechanism must be operative.

Escape of hydrogen poses no particular problem, even if the exospheric temperature is as low as $200^{\circ} \mathrm{K}$, a reasonable estimate for Io if thermospheric temperatures are maintained solely by absorption of ultraviolet solar radiation. Escape of nitrogen is more difficult. Thermal escape is vanishingly small for present purposes if we ignore the influence of Jupiter's gravitational field. The close proximity of Jupiter, however, leads to a major perturbation of the gravitational surfaces around Io, as illustrated in figure 1 . The net gravitational field passes through zero at a height of $770 \mathrm{~km}$ above the sub-Jupiter point on Io. Beyond this point material is no longer gravitationally bound to Io, although it must remain in orbit around Jupiter. The flux across the gravitational null surface is given, with sufficient accuracy for present purposes, by $n v$, 


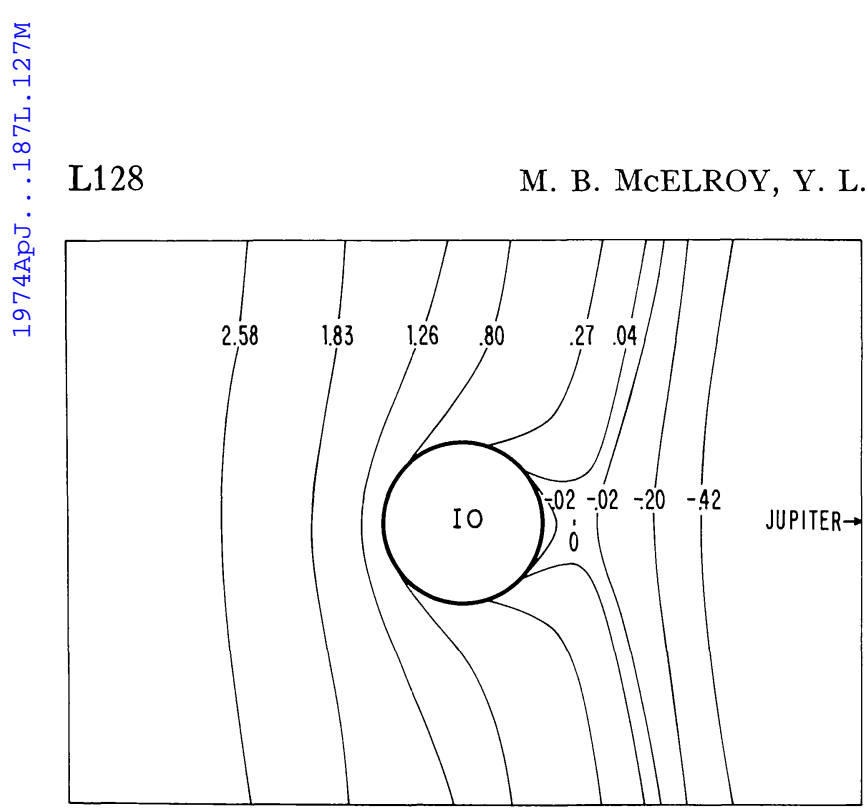

Frg. 1.-Gravitational equipotential surfaces in the vicinity of Io. The numbers give the magnitude of the potential defined by $\phi(r, \theta)=\left\{-1 / R-\frac{1}{2} R \cos \theta+C\right\}$ in units of $140 \mathrm{~cm}^{2} \mathrm{~s}^{-2}$, where $C$ is an arbitrary constant chosen such that $\phi=0$ at the level where the gravitational force equals zero $R$ defines the radial distance from Io in units of Io's radius; $\theta$ is the polar angle taken as zero in the direction of Jupiter. The surface of Io is indicated by the solid circle.

where $n$ denotes number density and $v$ is the appropriate mean thermal velocity. The flux of $\mathrm{N}_{2}$ is less than $10^{9}$ $\mathrm{cm}^{-2} \mathrm{~s}^{-1}$ if we consider an atmosphere at $200^{\circ} \mathrm{K}$. The flux of $\mathrm{N}$ could be significantly larger, although even in this case escape is not likely to proceed with sufficient rapidity to balance the photolytic source in the lower atmosphere.

The sodium observations suggest a possible solution to this dilemma. Energetic electrons, entering the atmosphere on occasions when the satellite is aurorally active, can lead to intense heating of the upper atmospheric regions. An energy source of $1.7 \times 10^{4}$ ergs $\mathrm{cm}^{-2}$ would suffice to raise a column of $\mathrm{N}_{2}$ with density $10^{16} \mathrm{~cm}^{-2}$ to a temperature of $8000^{\circ} \mathrm{K}$. A heat source of this magnitude, which could be provided by a plasma physical process such as that suggested by Goldreich and Lynden-Bell (1969) or by Gurnett (1972), would generate strong vertical motions, and gas would escape efficiently through the gravitational null surface. An energy source as small as $2 \mathrm{ergs} \mathrm{cm}^{-2} \mathrm{~s}^{-1}$, present for as little as 10 percent of the time, could account for the necessary $\mathrm{N}_{2}$ escape flux of $2 \times 10^{11}$ molecules $\mathrm{cm}^{-2} \mathrm{~s}^{-1}$.

\section{EXCITATION AND TRANSFER OF SODIUM RADIATION}

We propose that sodium atoms are excited primarily by collisions involving vibrationally excited $\mathrm{N}_{2}$,

$$
\mathrm{N}_{2}(v)+\mathrm{Na}\left({ }^{2} S\right) \rightarrow \mathrm{N}_{2}\left(v^{\prime}\right)+\mathrm{Na}\left({ }^{2} P\right) ;
$$

although there may be an additional contribution from

$$
\mathrm{N}_{2}\left(A^{3} \Sigma^{+}{ }_{u}\right)+\mathrm{Na}\left({ }^{2} S\right) \rightarrow \mathrm{N}_{2}+\mathrm{Na}\left({ }^{2} P\right),
$$

and from resonance scattering of sunlight in the toroidal ring. The vibrationally and electronically excited mole- cules in reactions (1) and (2) would be formed by collisional processes involving low-energy electrons during auroral conditions on Io.

Both reactions (1) and (2) are known to occur with high efficiency (Hurle 1964; Krause, Fricke, and Fite 1972; Gann, Kaufman, and Biondi, 1972; Sadowski, Schiff, and Chow 1972), and the effective temperature of the emitted D lines is large-greater than $1500^{\circ} \mathrm{K}$ even when the kinetic temperature is relatively low, $\sim 400^{\circ} \mathrm{K}$ (Dugan and Acker 1972; Gann et al. 1972). Reaction (1) was suggested earlier by Hunten (1965) to account for sodium emission in terrestrial aurorae. As much as 50 percent of the energy contained in the low-energy electrons assumed to enter Io's atmosphere could be emitted as radiation in the D lines, even under conditions where the sodium mixing ratio was as low as $10^{-3}$. A mixing ratio of this magnitude could provide an emission of $10^{3}$ kilorayleighs if the column density of $\mathrm{N}_{2}$ in the emitting layer were as large as $10^{16} \mathrm{~cm}^{-2}$.

The intensity of radiation at wavelength $\lambda$ in the $D$ lines is given by solution of the appropriate transfer equation. With standard notation, assuming a planeparallel medium, we have

$$
\mu \frac{d I_{\lambda}}{d \tau}=I_{\lambda}-\frac{1}{2} \int_{-1}^{1} I_{\lambda} d \mu-\epsilon_{\lambda},
$$

where $\epsilon_{\lambda}$ will be a function of $\tau$, and in fact it is necessary to make some explicit assumptions about this dependence in order to reproduce the observed intensity ratio, D2/D1. We consider a model in which excitation occurs in a hot atmospheric layer underlying a cooler scattering medium. The scattering medium is intended to describe the effects of sodium atoms in a gaseous envelope around Io.

We denote the optical thickness of the scattering medium by

$$
\tau(\lambda)=\tau_{0} \exp \left[-\left(\frac{\lambda-\lambda_{0}}{\Delta \lambda}\right)^{2}\right]
$$

where

$$
\Delta \lambda=\lambda_{0} c^{-1}\left(2 k T_{c} / M\right)^{1 / 2}
$$

and $\tau_{0}$ defines the optical thickness at the line center, $\lambda_{0}$. The thickness of the emitting layer is given by

$$
\Delta \tau(\lambda)=\Delta \tau_{0} \exp \left[-\rho^{2}\left(\frac{\lambda-\lambda_{0}}{\Delta \lambda}\right)^{2}\right],
$$

with

$$
\rho=\left(T_{c} / T_{h}\right)^{1 / 2}
$$

where $T_{c}$ and $T_{h}$ are the temperatures for the scattering and emitting layers, respectively. We take

$$
\epsilon_{\lambda}=\epsilon_{0} \exp \left[-\rho^{2}\left(\frac{\lambda-\lambda_{0}}{\Delta \lambda}\right)^{2}\right]
$$

in the emitting layer and set the collisional source term, $\epsilon_{\lambda}$, equal to zero elsewhere. 
With these assumptions, using a two-stream approximation to solve equation (3), we find

$I_{1}(\lambda)=\frac{3^{1 / 2} \epsilon_{\lambda} \Delta \tau(\lambda)\left[2(1+r)+(1-r) 3^{1 / 2} \Delta \tau(\lambda)\right]}{\left\{2+(1-r) 3^{1 / 2}[\Delta \tau(\lambda)+\tau(\lambda)]\right\}}$.

Here $I_{1}(\lambda)$ denotes the emergent intensity at wavelength $\lambda$ in the D1 line, and $r$ defines the surface albedo of Io which we take to be independent of $\lambda$ for purposes of the present application. The intensity at wavelength $\lambda$ in the D2 line is simply obtained by doubling the appropriate values of $\Delta \tau$ and $\tau$ in equation (9).

Figure 2 shows the computed profiles of the emergent D1 and D2 lines. These profiles were derived with a reasonable choice for the parameters $\tau_{0}, \Delta \tau_{0}, T_{c}$, and $T_{h}$, and the calculated intensity ratio, $\mathrm{D} 2 / \mathrm{D} 1$, is consistent with observation. The reduction in D2, as illustrated in figure 2, occurs mainly at the expense of photons near the line center. These photons are effciently trapped in the lower atmosphere and are therefore preferentially absorbed at Io's surface.

We also investigated models in which the radiative source term $\epsilon_{\lambda}$ was assumed independent of $\tau$ and models in which the radiative source was assumed to occur above a scattering medium. Intensity ratios, $\mathrm{D} 2 / \mathrm{D} 1$, for these models are typically in the range 1.8 to 2.0 , in contrast to observed values in the range 1.2 to 1.6. We conclude that the observed intensity ratio places strong constraints on theoretical models for the luminosity. It seems difficult to escape the conclusion that sodium radiation from Io must be strongly scattered in an extended envelope ejected by Io during periods of auroral activity. The values of $\tau_{0}$ and $\Delta \tau_{0}$ required to account for different values of the intensity ratio D2/D1 are summarized in figure 3 . If we select a particular value for this ratio and a particular value for

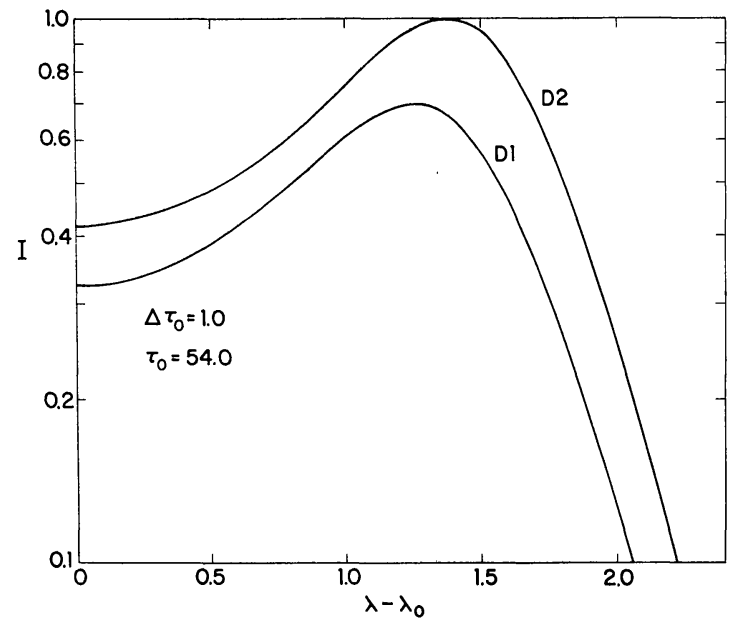

FIg. 2.-Computed profiles for the D1 and D2 lines with $\Delta \tau_{0}=$ 1.0 and $\tau_{0}=54.0$. The reflectivity of Io is taken as 0.4 , and $\rho$ is equal to 0.7 . The wavelength scale $\left(\lambda-\lambda_{0}\right)$ is in units of the Doppler width in the hot layer. Intensity is given in arbitrary units, but a common scale is used for both lines.

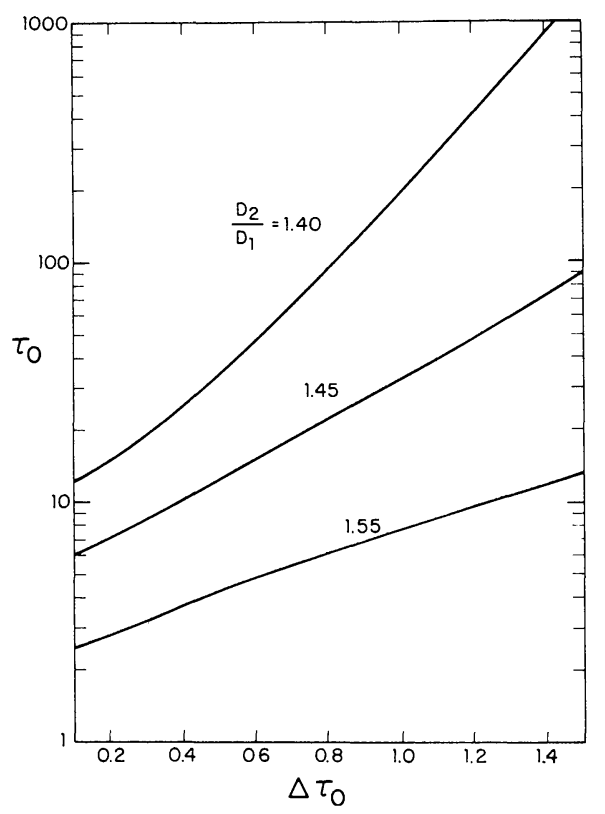

Fig. 3.- Values of $\tau_{0}$ and $\Delta \tau_{0}$ consistent with various ratios $\mathrm{D} 2 / \mathrm{D} 1$. The transfer model assumes $r=0.4$ and $\rho=0.7$ as in fig. 2 .

either $\tau_{0}$ or $\Delta \tau_{0}$, then high-resolution observations of the emission can be used to compute $T_{h}$. The range of values for $T_{h}$ consistent with the observations of Brown and Chaffee (1974) is shown in figure 4.

\section{DISCUSSION}

The model developed above has a number of implications, several of which are subject to direct observational check. There should be an extensive cloud of

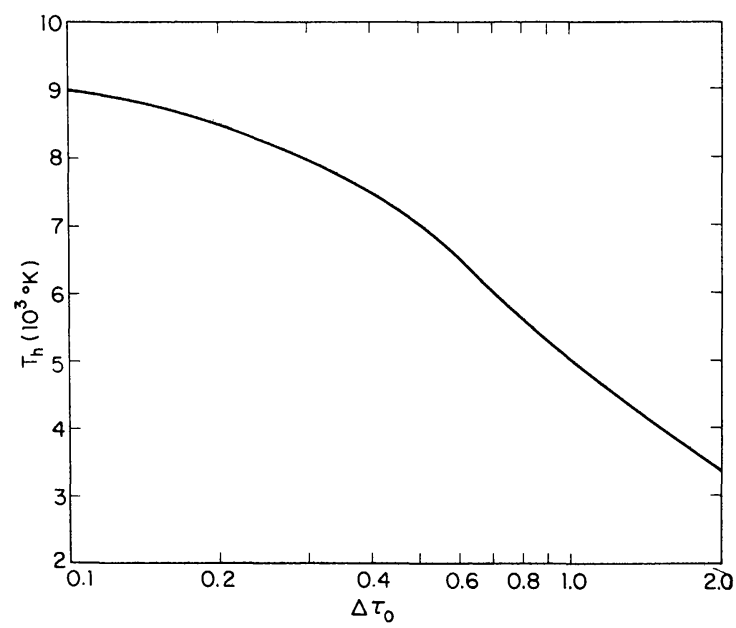

Fig. 4.-Values for the temperature in the hot emitting layer of Io's atmosphere required to account for line profiles observed by Brown and Chaffee (1974), as functions of $\Delta \tau_{0}$. 
nitrogen, hydrogen, sodium, and perhaps other gases, around Jupiter in the vicinity of Io's orbit, similar to the toroidal ring discussed by $\mathrm{McD}$ Donough and Brice (1973) for Titan. If the lifetime of gas in the cloud is determined by photoionization, the cloud could contain $10^{36}$ nitrogen molecules, $10^{37}$ hydrogen molecules, a somewhat smaller number of hydrogen atoms, and perhaps as many as $10^{31}$ sodium atoms. The hydrogen cloud should be detectable with instrumentation carried by Pioneer 10, and may indeed have been observed already by Moos, Fastie, and Bottema (1969) who found a surprisingly large intensity from Jupiter at L- $\alpha$. Gas in the cloud, after it is ionized, would be swept up by Jupiter's magnetic field and could form a significant source of trapped magnetospheric plasma.

There should be a detectable ionosphere on Io, formed by photoionization of sodium. If we assume that sodium ions are removed mainly by radiative recombination, we find $n_{e}^{2} \approx 10^{5}[\mathrm{Na}]$, where $n_{e}$ denotes electron density $\left(\mathrm{cm}^{-3}\right)$ and $[\mathrm{Na}]$ denotes the density of sodium atoms in similar units.
The escape rates considered here imply significant loss of surface ice over geologic time. We estimate that a layer of thickness $10 \mathrm{~km}$ over the entire surface of Io could be depleted in $5 \times 10^{9}$ years.

The model also has implications for other Galilean satellites of Jupiter. We would expect escape processes to be less efficient for these bodies; and it is worth noting that one of them, Ganymede, has a detectable atmosphere (Carlson et al. 1973). If Ganymede had a significant layer of surface $\mathrm{NH}_{3}$ ice, we might expect $\mathrm{N}_{2}$ as an abundant atmospheric gas. Photolysis of $\mathrm{H}_{2} \mathrm{O}$, in equilibrium with surface water ice (Pilcher, Ridgway, and McCord 1972), could also lead to a source of atmospheric $\mathrm{O}_{2}$. It seems probable that Ganymede, like Io, should be surrounded by a toroidal ring. The thickness of Ganymede's ring, however, is most probably less than that for Io.

This research was funded by the Atmospheric Sciences Section of the National Science Foundation under grant GA-33990X to Harvard University.

\section{REFERENCES}

Binder, A. B., and Cruikshank, D. P. 1964, Icarus, 3, 299-305.

Bosch, E. 1954, Zs. f. Phys., 137, 89-103.

Brown, R. A. 1973, IAU. Symposium No. 65, in press.

Brown, R. A., and Chaffee, F. H. 1974, A p. J. (Letters), 187, L125.

Carlson, R. W., Bhattacharyya, J. C., Smith, B. A., Johnson,

T. V., Hidayat, B., Smith, S. A., Taylor, G. E., O'Leary, B., and Brinkmann, R. T. 1973, Science, 182, 53.

Cruikshank, D. P., and Murphy, R. E. 1973, Icarus, in press.

Dugan, C. H., and Acker, B. D. 1972, Chem. Phys. Letters, 14, 2, 190-192.

Gann, R. G., Kaufman, F., and Biondi, M. A. 1972, Chem. Phys. Letters, 16, 2, 380

Goldreich, P., and Lynden-Bell, D. 1969, Ap. J., 156, 59.

Gurnett, Donald A. 1972, Ap.J., 175, 525.

Hunten, D. M. 1965, J. Atm. Terr. Phys., 27, 583-586.

Hurle, I. R. 1964, J. Chem. Phys., 41, 3911.
Hurley, I., Tuttle, Jr., T. R., and Golden, S. 1968, J. Chem. Phys., $48,6,2818-2819$.

Johnson, T. V., and McCord, T. B. 1972, Icarus, 13, 37-42.

Krause, H. F., Fricke, J., and Fite, W. L. 1972, J. Chem. Phys., 56, 9, 4593 .

Kuiper, G. P. 1973, Sky and Tel. (Oct.) p. 228.

McDonough, T. R., and Brice, N. M. 1973, Nature, 242, 513.

Moos, H. W., Fastie, W. G., and Bottema, M. 1969, A p. J., 155, 887.

Morrison, D., and Cruikshank, D. P. 1973, Icarus, 18, 224-236.

Pilcher, C. B., Ridgway, S. T., and McCord, T. B. 1972, Science, 178, $1087-1089$.

Sadowski, C. M., Schiff, H. I., and Chow, G. K. 1972, J. Photochem., 1, 23

Sinton, W. M. 1973, Icarus, in press.

Smith, B. A., and Smith, S. A. 1972, Icarus, 17, 218-222.

Wildt, R. 1939, M.N.R.A.S., 99, 616-623. 